\title{
EVALUATION OF IMPLANT ASSISTED PROSTHESIS RESTORING FLABBY MANDIBULAR RIDGE (CBCT STUDY)
}

\author{
Faten A.S. Abu Taleb*
}

\begin{abstract}
Background: The poor quality denture bearing area caused by displaceable flabby tissue is one of the soft tissue abnormalities that commonly encountered in the clinical practice. Surgically or non-surgically, techniques have suggested circumventing this difficulty.

Aim: This study aimed to evaluate the management of anterior flabby tissues of completely edentulous mandible using dental implant with either conventional functional impression or selective pressure impression technique.

Methods: Twelve completely edentulous patients with anterior mandibular flabby tissues were included in this prospective clinical study. All patients received upper complete denture and lower complete implant assisted overdenture constructed over two implants, placed at the canine region. Patients were randomly divided into two groups. Group I, patients received their overdenture constructed from conventional functional impression technique, while patients of group II received the overdenture constructed from selective pressure impression technique. All patients underwent cone-beam computed tomography scanning at insertion, six and twelve months to measure the amount of marginal bone loss around the implants and the thickness of anterior mucosa between the two implants. All data are collected, tabulated and statistically analyzed.
\end{abstract}

Result: dentures constructed over dental implant for the two groups resulted in marginal bone loss around implant and decrease in flabby tissue thickness at all follow up period at denture insertion, six months and twelve months. When comparing between the two groups at the same follow up period, the patients receiving dentures constructed with selective pressure impression technique resulted in less marginal bone loss and more decrease in soft tissues thickness than those dentures constructed with conventional functional impression techniques

Conclusion: Using dental implant is recommended as a way for flabby tissues management. While, using implant with selective pressure impression technique is an effective manner for flabby tissues management than using dental implant alone.

KEYWORDS: Displaceable tissues- Implant retained overdenture- Selective pressure impression technique- Cone Beam Computed Tomography.

* Assistant Prof of Removable Prosthodontics, Prosthodontic Department, Faculty of Dentistry, Tanta University. 


\section{INTRODUCTION}

Poor retention and stability, continued bone loss, and decrease of confidence in dental treatments are common complains especially if there are flabby tissues. Flabby ridge is defined as an excessive movable tissue ${ }^{1}$. Development of flabby ridge has been caused by one or more following factors as atrophy, bone resorption, nutritional deficiencies, pressure, unplanned and uncontrolled dental extraction, functional forces and patients with parafunctional habits ${ }^{2,3}$. Flabby ridges are composed of mucosal hyperplasia and loosely arranged fibrous connective tissue as well as dense collagenase connective tissue ${ }^{4}$. In the edentulous patient, it is commonly found in the anterior region in both arches. This problem is more pronounced in the mandibular arch, due to less available tissue surface $^{5}$.

Massad and Lobel $^{6}$ have graded the displaceability of flabby tissues as low, average clinically acceptable and high displaceability and the last condition is very difficult to treat. The thickness and mobility of mucosa over the ridges in completely edentulous patients is variable. In some areas, the thickness ranges from 2 to $4 \mathrm{~mm}$. In other areas where the atrophy of the alveolar process was rapid and sophisticated, the mucosa has no bone support and becomes loose and flabby. Such mucosa is more than $4 \mathrm{~mm}^{\text {thick }}{ }^{7}$. Concerns about support for the denture should be noted if the residual ridge mucosa moves, more than $2 \mathrm{~mm}$ under light pressure ${ }^{8}$.

Effective management of a patient with flabby ridge areas may include one or a combination of more than one treatment modality, such as surgical removal of flabby tissue, conventional prosthodontics measures without surgical intervention and implantsupported prosthesis ${ }^{9,}{ }^{10}$. Flabby ridges can be managed surgically by excision, ridge augmentation, and injection of sclerosing solutions. The advantage of this approach is that a firm denture-bearing area is produced, which enhances the stability of the prosthesis ${ }^{11}$, but the health of the patient must be taken into consideration and removal of these tissues is contraindicated in circumstances where little or no alveolar bone remains. It can be argued however that the fibrous part of the ridge has a cushioning effect that reduces trauma to the underlying bone and therefore should not be removed. Retention also adversely affected by the significant loss of the sulcus depth that is important in aiding border seal ${ }^{12}$.

When considering conventional prosthodontics, a special care and attention are needed during the recording of the tissue, because forces exerted during this act can result in distortion of the mobile tissue and denture instability. Several impression techniques are used for management of flabby ridge as one part impression technique (Selective perforation tray), controlled lateral pressure technique, palatal splinting using a two-part tray system, selective composition flaming, two parts impression technique, and mucostatic and mucodisplacive combination ${ }^{13}$. Various unconventional approaches as fluid retained denture, the concept of neutral zone $^{14}$ and internally weighted mandibular complete denture are advocated with flabby ridge ${ }^{9}$.

A problem is encountered in the conventional impression making if a flabby area is present within an otherwise "normal" denture bearing area. If these tissues are compressed during conventional impression making it will later tend to recoil and dislodge the overlying denture ${ }^{15}$. Thus, over the years, several impression techniques have been suggested for the impression of a flabby tissue ridge that will support the flabby tissue but at the same time will not displace it. Selective pressure or minimally displacive impression techniques overcome these limitations. The use of relief holes, windows and wax relief reduces the hydraulic pressure and minimizes the displacement of the hypermobile tissues ${ }^{16,17}$.

Although the mandible suffers a greater magnitude of resorption, the anterior mandible widens toward the inferior border. The absence 
of a mandibular residual ridge does not influence the use of implants since the width and depth of basal bone below the floor of the mouth is usually substantial with a prominent inferior cortex ${ }^{18}$. Two Implant retained mucosal-supported overdenture is proposed to be the first line of treatment for edentulous mandible patients ${ }^{19}$.

Many authors studied the factors that thought to have an impact on bone level after implant installation as surgical trauma,overload, microgap polished implant neck, peri-implantitis, prosthetic abutment material and its manipulations ${ }^{20}$. Moreover, as mentioned by Berglundh et $\mathrm{a}^{21}$ the influence of mucosal thickness and biological width formation that serves as a protective mechanism for underlying bone is not so meticulously analyzed as other factors. It has been proposed that a minimum $3 \mathrm{~mm}$ of peri-implant mucosa is required for the stable epithelial connective tissue attachment to form without bone loss. There are some suggestions that if minimal dimension of mucosal tissues is not available, bone loss may occur to ensure the proper development of biological width ${ }^{22}$. The formation of peri-implant soft tissues after implant installation is a complex process, which affected by the characteristics of mucosal tissues and there is a relationship among mucosal thickness and marginal bone loss around implants ${ }^{21}$.

Masticatory mucosal thickness was measured using several methods. Physical measurement methods were invasive methods that used needles and periodontal probes after local anesthetic administration. Ultrasonic devices were noninvasive and easy to use, however, this method lacked consistency ${ }^{23}$. Measurements of mucosal thickness have been recorded beneath dentures' fitting surfaces by using a central bearing device known as tilt meter to measure the thickness the mucosa by assessing denture movement during function. Later a kinesographic instrument was used to determine denture movement and thus mucosal thickness ${ }^{24}$. In a recent retrospective study, conebeam computed tomography (CBCT) with lowdose radiation has been explored as a non-invasive technique which provided an accurate assessment of mucosal tissue thickness ${ }^{25}, 26$. The results were very similar to previous reports that employed physical measurements 23-26. This led to the hypothesis that CBCT could be applied for visualizing and measuring soft tissues thickness ${ }^{27-28}$.

Prosthodontic literature has documented various treatment modalities for overcoming the problem of the flabby ridge but little researches have seen about the use of dental implant in management of flabby ridge. The aim of this study was to evaluate the using of dental implant with either conventional functional impression or selective pressure impression technique will be considered better for flabby tissue management.

The hypothesis : Dental implant alone is a management way for flabby ridge.

\section{MATERIALS AND METHODS}

\section{Patient's selection}

Twelve completely edentulous male patients aged between 55 to 65 years complained from previous unretentive and unstable lower complete denture overlying residual alveolar ridge with anterior flabby tissues were selected from the outpatient clinic of the Prosthodontics Department, Faculty of dentistry Tanta University. The patients with realistic expectations about esthetic and retentive outcome of implant therapy. Patients should had normal ridge relationship (Skeletal Angle's class I maxillomandibular relationship) and adequate inter-arch space. All selected patients had adequate height, width and quality of bone. Patients should had no history of habits that might jeopardize ossteointegration process such as heavy smokers and alcoholism, catabolic drugs or psychiatric disorders and able to undergo minor oral 
surgery. Patient should be free from neuromuscular or tempromandibular joint disorders and no history of chemotherapy or radiotherapy. The patients were motivated to follow proper oral hygiene measures.

All patients were instructed to sign a Medicolegal written consent with detailed information about the Surgical, Prosthetic, Radiographic, Photographic and Laboratory procedure, the possible postoperative complications and implant failures, the recall visits for follow-up and the parameters that would be assessed

\section{Surgical procedure}

Clinical examination of the mucosa covering the residual ridge to ensure that the mucosa was free from signs of inflammation, infection, or irritation. Also, to detect presence of flabby tissues and its thickness (Figure 1).

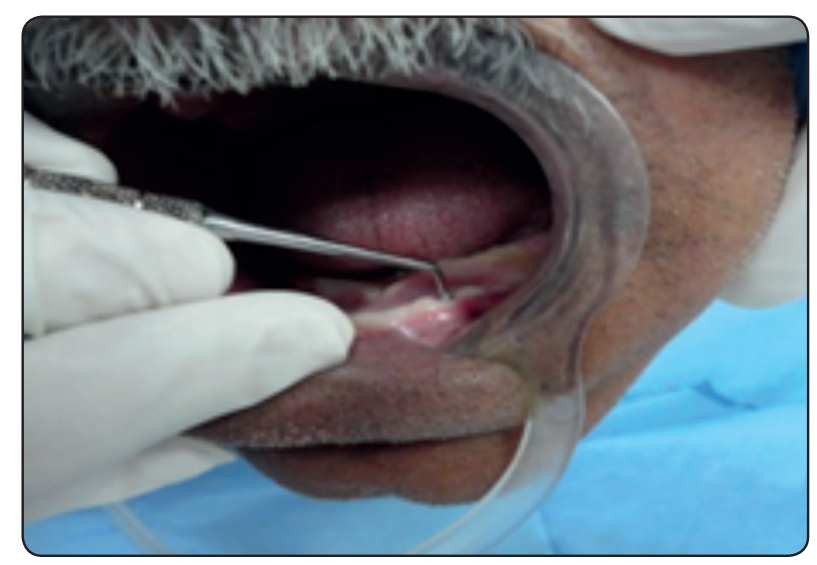

Fig. (1) The clinical identification of flabby tissue

A new denture was made for all patients and a transparent acrylic resin surgical stent was made from a denture duplicate and two holes were done in the canine region. The dental implant (Dentium Implant System Korea)with a diameter of $4.3 \mathrm{~mm}$ and a length of $14 \mathrm{~mm}$ were placed bilaterally in the canine region of the mandible

\section{Prosthetic procedure}

After three months to ensure complete ossteointegration, the healing abutments
(FRIADENT, Ball and Socket Attachment) were determined according to the mucosal thickness overlying the implants, the available inter-arch space and over denture thickness and were placed on the implant. Patients participating in this study were randomly divided into two equal groups, patients of the first group received mandibular complete overdenture constructed from conventional functional impression technique with opposing conventional complete maxillary denture. While patients in the second group received mandibular complete overdenture constructed from selective pressure impression technique with opposing conventional complete maxillary denture.

\section{Selective pressure impression technique ${ }^{29}$}

The displaceable tissues identified on the mandibular cast that obtained from primary impression, and then a layer of wax (Kerr base plate wax Kerr Italia Italy) was adapted on the flabby area to provide extra relief in the flabby region followed by addition of one more layer of wax covering the ridge except the buccal shelf area. A mandibular custom tray was fabricated with autopolymerising clear acrylic resin. The buccal shelf area was recorded by using mucocompressive impression material like impression compound Type 1 (Kerr Impression compound Kerr Italia Italy). The remaining borders of tray were recorded by using green stick compound (Verde/Green, Curupayti, Argentina). The spacer wax was then removed and multiple holes were drilled in the region of the flabby tissue and tray adhesive was applied. A final impression with monophase (medium body) (Aquasil LV Monophase, Dentsply Caulk) was carried (Figure 2). Subsequently, conventional treatment procedures were followed to deliver a complete over denture.

\section{Evaluation of marginal bone loss around implant}

Using the linear measurement system supplied by the -CBCT (K.V.P.85, M.A.16, Field of view 7 $\mathrm{cm} \times 14.5 \mathrm{~cm} \times 14.5 \mathrm{~cm}$ ) this was done at denture 
insertion, after 6, and 12 months post-insertion. Aligning the patient: For correct alignment, the trans axial CT slice plane was parallel to the occlusal plane of the upper jaw. The relationship of the jaws was stabilized during the scan using a radiolucent silicone bite registration index.

To evaluate vertical bone levels around the implants. The marginal bone height was determined at the mesial, distal, buccal, and lingual sites of each implant using software (blue sky software, IMTEC Imaging) by measuring the distance from the implant apex to the first visible bone-to-implant contact. The first bone-to-implant contact at surgery was defined as baseline. The labial and lingual bone heights were measured on the sagittal view screen, while the mesial and distal bone heights are measured on the coronal view screen. The mean value of readings of mesial,distal, buccal, and lingual together was taken, tabulated and statistically analyzed (Figure 3).

\section{Thickness of flabby tissues ${ }^{24}$ :}

A radiopaque Poly (methyl methacrylate (AngloEgyptian Company. Hegaz, Cairo, Egypt, Batch No.505/04) and Barium Titanate ((Acros, USA) denture duplicate was constructed to visualize the mucosa and separate the soft tissue of the lips, cheeks, and tongue away from the surface of alveolar mucosa in the mouth. At each measurement the fitting surface of the denture duplicate was lined with a thin layer of alginate impression material (Alginate impression material, Cavex CA 37, Cavex, Netherlands. Holland) mixtured with barium

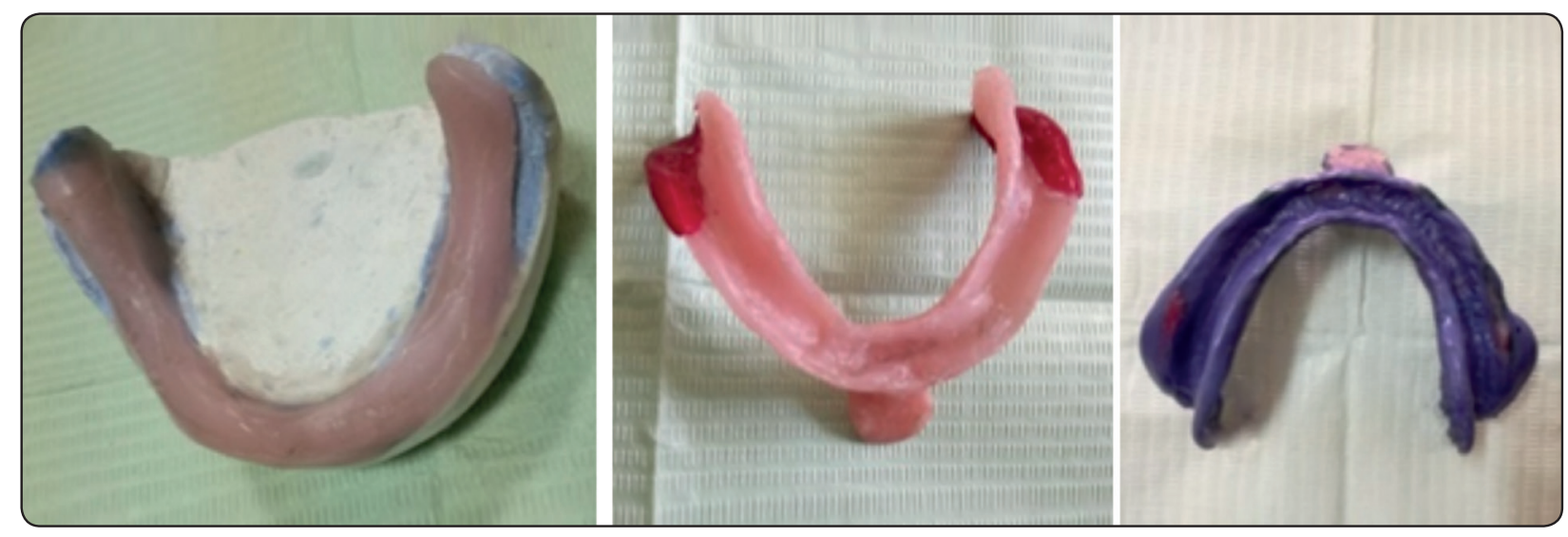

Fig. (2) The steps of selective pressure impression technique of mandibular arch.

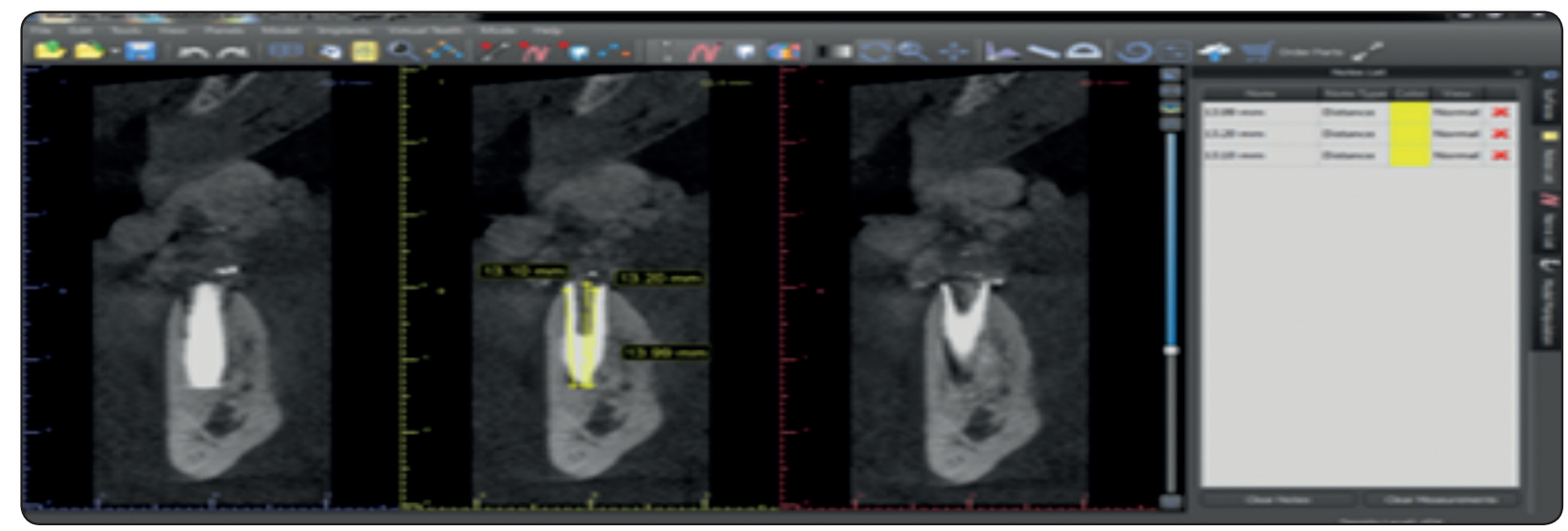

Fig. (3) Cone beam computed tomography image demonstrating the marginal bone loss 
titanate $1: 2$ by weight, and was allowed for an intraoral setting time of 3 minutes. With the denture duplicate and impression mix in place, a scan was obtained with the CBCT machine. The relationship of the jaws was stabilized during the scan using a radiolucent silicone bite registration index. The scan radiation was adjusted at $90 \mathrm{KV}$ and $8 \mathrm{~mA}$. This allowed the patient's bone to appear on the scan. The cross-section CBCT images perpendicular to the alveolar ridge crest lines of the mandible in the buccolingual direction were extracted with the CBCT software (Planmeca Romexis, Planmeca Group, Finland) from patients.

\section{Measurements}

On the reconstructed images of the denture duplicate in place, the area of the alveolar mucosa between the denture duplicate with its lining impression and the alveolar bone was measured at three equidistant points between the two implants for each image. The average of these areas were measured and recorded at insertion, at 6 and 12 months.(Figure 4).

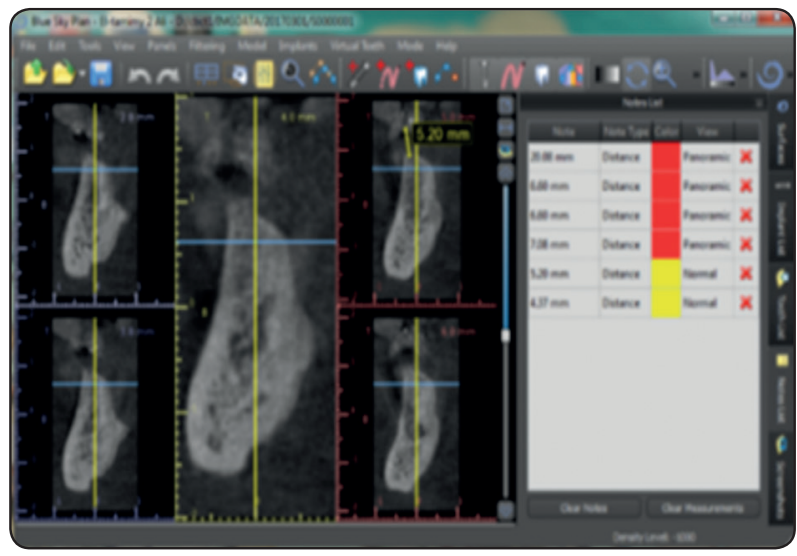

Fig. (4) Cone beam image showed the measurement of soft tissue thickness between the two implants

\section{RESULTS}

The collected data was organized tabulated, and statistically analyzed using both of statistical package for the social sciences (SPSS version 23; SPSS INC., Chicago, Illinois, USA) Software and
MedCalc ${ }^{\circledR}$ Version 15.6.1-64-bit- copyright $\mathbb{C}$ 1993-2015. For quantitative variables, mean and SD were calculated. Using the KolmogorovSmirnov test (Normality Test) to determine the type of testing used whether it is a parametric test such as independent $t$ test and one way ANOVA or non-parametric test such as Mann-Whitney (U) test and Friedman test. The difference within the same group in relation to different periods of follow-up as compared with baselines was done using the MannWhitney $\mathrm{U}$ is non-parametric test which equivalent independent samples t-test. Also, the difference between groups was statistically analyzed using the Friedman test is non-parametric test which equivalent one way ANOVA.

\section{Marginal bone loss:}

The mean and standard deviation of marginal bone loss (MBL) at different observation times for both groups was shown in table 1 and figure 5 . There was significant difference in MBL at six and at twelve months of measurements for both groups $(\mathrm{P}=.0 .0001$ for group 1 and 0.00001 for group 2$)$

In comparing between the two groups, There was significant difference at the different follow up periods. The second group showed less bone resorption than the first group as showed in table 2 and figure 6,7. $(\mathrm{P}=0.002$ and 0.001 at six and twelve months respectively)

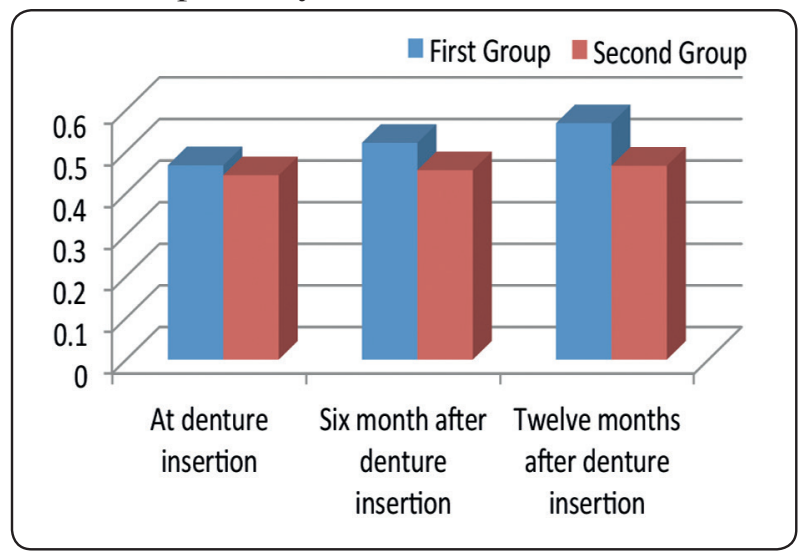

Fig. (5) average marginal bone loss implant of the between groups during the follow up period 
TABLE (1) Showed comparison of marginal bone loss of both groups during the follow up period

\begin{tabular}{|l|c|c|c|c|c|c|}
\hline \multirow{2}{*}{ Marginal bone loss } & \multicolumn{3}{|c|}{ Group 1 } & \multicolumn{3}{c|}{ Group 2 } \\
\cline { 2 - 7 } & Chi-squared & DF1/DF2 & P value & Chi-squared & DF1/DF2 & P value \\
\hline At denture insertion & & & & & \\
\cline { 1 - 4 } At six months & 24.0000 & $2 / 22$ & $0.0001^{* *}$ & 292.6000 & $2 / 22$ & $.000001^{* *}$ \\
\cline { 1 - 4 } At twelve months & & & & & \\
\hline
\end{tabular}

TABLE (2) Comparison of marginal bone loss between the two groups at the different follow up periods

\begin{tabular}{|c|c|c|c|c|c|c|c|c|c|c|}
\hline \multirow{3}{*}{ Marginal bone loss } & \multicolumn{4}{|c|}{ Group 1} & \multicolumn{4}{|c|}{ Group 2} & \multirow{3}{*}{$\begin{array}{c}\text { Mann- } \\
\text { Whitney } \\
\text { U }\end{array}$} & \multirow{3}{*}{$P$ value } \\
\hline & \multicolumn{2}{|c|}{ Right implant } & \multicolumn{2}{|c|}{ Left implant } & \multicolumn{2}{|c|}{ Right implant } & \multicolumn{2}{|c|}{ Left implant } & & \\
\hline & Mean & SD & Mean & SD & Mean & SD & Mean & SD & & \\
\hline At implant insertion & 0 & 0 & 0 & 0 & 0 & 0 & 0 & 0 & ---- & --- \\
\hline At denture insertion & 0.471 & 0.04699 & 0.463 & 0.04003 & 0.440 & 0.04431 & 0.446 & 0.03655 & 20.00 & 0.0853 \\
\hline At six months & 0.518 & 0.05937 & 0.523 & 0.05565 & 0.454 & 0.04795 & 0.456 & 0.04033 & 18.00 & $0.0027^{* *}$ \\
\hline At twelve months & 0.562 & 0.07213 & 0.574 & 0.06775 & 0.464 & 0.05275 & 0.468 & 0.04282 & 42.50 & $0.0018^{* *}$ \\
\hline
\end{tabular}

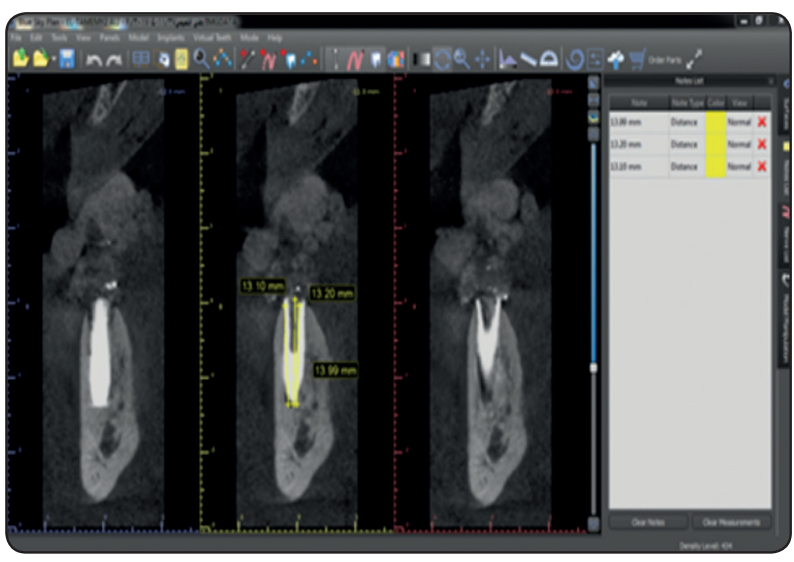

Fig. (6) Cone beam image showed the amount of marginal bone loss around implant in the first group at twelve months.

\section{Thickness of flabby tissues:}

The mean and standard deviation of soft tissues thickness at different observation times for both groups was shown in table 3 and figure $8,9,10$. There was significant difference at six and at twelve months of measurements for both groups (Independent samples t-test, $\mathrm{P}<.05$ ).

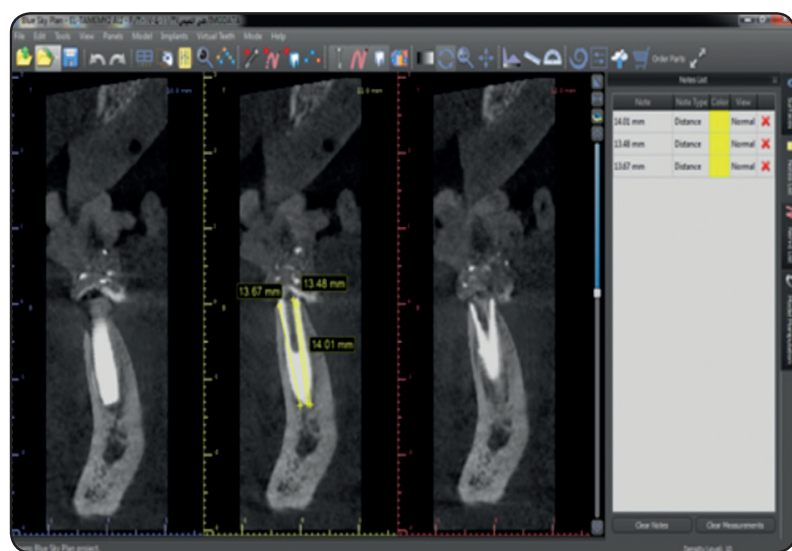

Fig. (7) Cone beam image showed the amount of buccolingual marginal bone loss around implant in the second group at twelve months

In comparing between the two groups, There was significant difference at the different follow up periods $(\mathrm{p}<0.05)$ as showed in table 4 . Where Friedman test is non-parametric test which equivalent one way ANOVA. 
TABLE (3) Showed the soft tissue thickness of the two groups during the follow up period using

\begin{tabular}{|l|c|c|c|c|c|c|}
\hline \multirow{2}{*}{ Soft tissue thickness } & \multicolumn{3}{|c|}{ Group 1 } & \multicolumn{3}{c|}{ Group 2 } \\
\cline { 4 - 7 } & F-test & DF1/DF2 & P value & F-test & DF1/DF2 & P value \\
\hline At implant insertion & & & & & & \\
\cline { 1 - 1 } At denture insertion & \multirow{2}{*}{5.222} & $3 / 44$ & $0.004^{* *}$ & 23.419 & $3 / 44$ & $0.001^{* *}$ \\
\cline { 1 - 3 } At six months & & & & & & \\
\hline At twelve months & & & & & & \\
\hline
\end{tabular}

TABLE (4) Comparison of soft tissue thickness between at different follow-up periods for each group

\begin{tabular}{|l|c|c|c|c|c|c|}
\hline \multirow{2}{*}{ Soft tissue thickness } & \multicolumn{2}{|c|}{ Group 1 } & \multicolumn{2}{c|}{ Group 2 } & Independent & \multirow{2}{*}{ P value } \\
\cline { 2 - 6 } & Mean & SD & Mean & SD & 0.311 & 0.7587 \\
\hline At implant insertion & 5.381 & 0.3643 & 5.425 & 0.3306 & 1.760 & 0.0923 \\
\hline At denture insertion & 4.892 & 0.2937 & 5.100 & 0.2860 & -2.229 & $0.0324^{* * *}$ \\
\hline At six months & 4.947 & 0.3891 & 4.700 & 0.2629 & -2.497 & $0.0205^{* *}$ \\
\hline At twelve months & 4.872 & 0.4036 & 4.517 & 0.2823 & \\
\hline
\end{tabular}

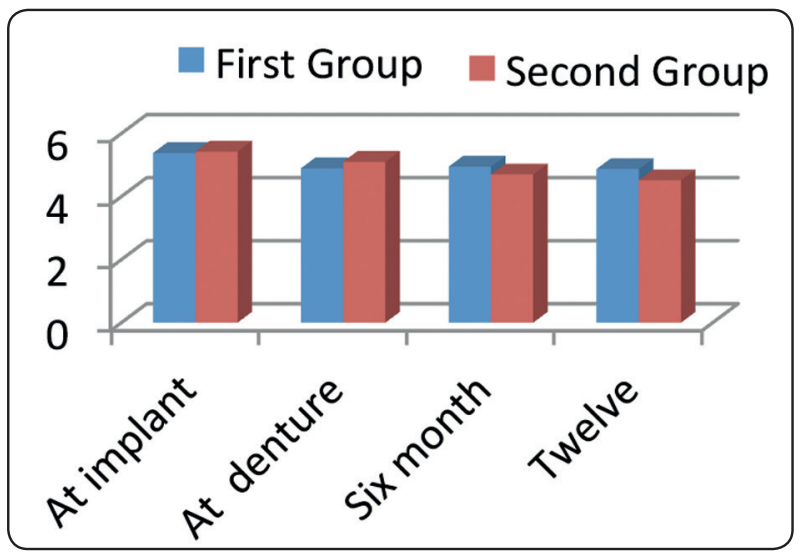

Fig (8) Average soft tissue thickness of the between groups during the follow up period

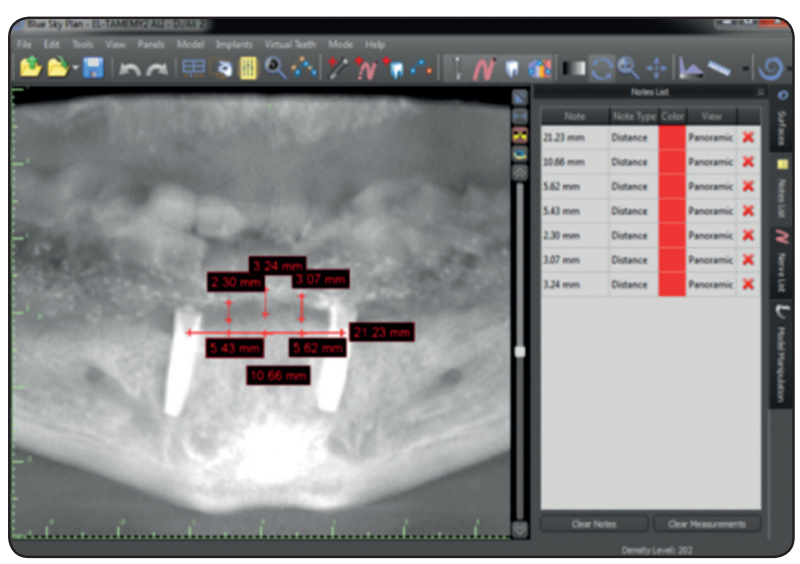

Fig. (10) Panoramic view of cone beam showed the decrease soft tissue thickness of the second group

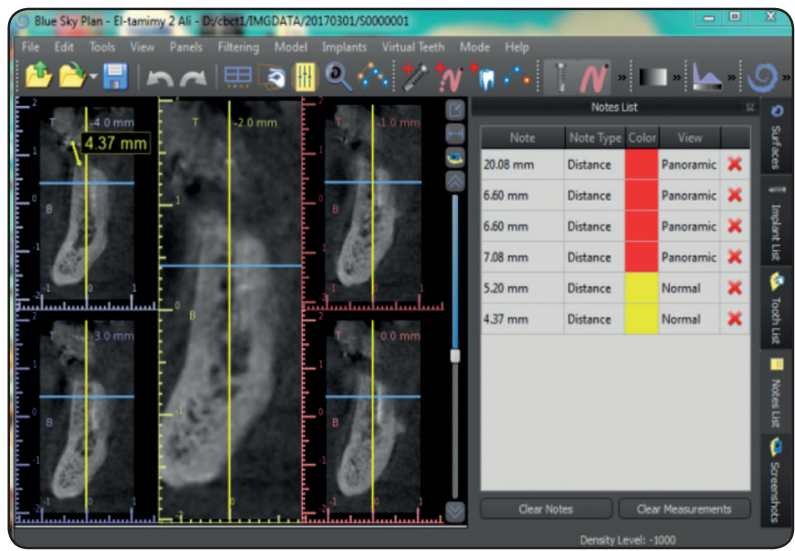

Fig. (9) Axial view of cone beam showed the decrease soft tissue thickness of the first group

\section{DISCUSSION}

Retention and support are reflection of the complete denture performance. Vohra ${ }^{19}$ and Jayaprakash et $\mathrm{al}^{30}$ stated that, the mandibular complete denture is unstable and poorly retentive due to low surface area. The resorbed alveolar bone is replaced by hyperplastic soft tissue and a flabby ridge is developed which was $13 \%$ to $26 \%$ and is commonly seen over the anterior part of the jaw. These movable tissues are easily displaced under 
masticatory forces, resulting in loss of peripheral seal and denture mobility with subsequently poor function, stability and discomfort that require relines/ rebase or remake of the prosthesis.

As reported with Lynch and Allen ${ }^{2}$, a common approach to solving a 'loose' complete denture is to apply some chair sides reline material. This approach is inconvenient, as the complete denture will act as a custom tray and with the reline material will advocate more tissue displacement. The tissues will once again tend to rebounded and the denture will still be 'loose.

Surgical approach may be contraindicated owing to the actuality that many complete denture patients are elderly or have complex medical history and the excision of flabby tissues will result in 'shallow' ridge that may affect the retention or resistance to lateral forces on the resultant denture. Although Bone augmentation is a remedy for this problem, but such treatment would be questionable. As it has the risk of rebuff of graft material and may need another surgery for graft ${ }^{31}$.

$\operatorname{Arora}^{32}$ stated that, the supposition behind the liquid supported denture as a treatment for flabby ridge is that, in the absence of masticatory force, the base undertakes its previous form that is the one during processing. Nevertheless, under masticatory load, the base adapts to the changed form of mucosa due to hydrodynamics of the liquid which improving retention, support, and stability. Optimal stress distribution of masticatory forces over a larger area and reduction of tissue overloading will result. However, this need thickness of denture base at least $3 \mathrm{~mm}$. and should be perfect and frequently checked for micro leakage.

There is an argument as to whether a pressure with mucodisplacive technique aiming to achieve maximum support or a mucostatic technique with the aim of achieving maximum retention should be employed. In the present study and with agreement of Chaturvedi et al ${ }^{13}$ the use of impression compound over the buccal shelf area for recording impressions ensures a stable and uniform contact on the buccal shelf area, which in this case is the primary stress bearing area. It also acts as a stopper for the tray in the final impression procedure. The use of light bodied elastomeric impression material instead of impression plaster because of the simple handling and manipulation of light viscosity elastomer over plaster.

As documented by Scarfe et al ${ }^{33}$, Ghoneima and $\mathrm{Kula}^{34}$ and Abdulhamid et al ${ }^{35}$, cone beam computed tomography CBCT images is painless way to obtain images in dentistry superior to panoramic images for identification of important nerves. Furthermore, the aspect ratio of the images obtained by CBCT was 1:1 and these images could be saved so periodic measurements were possible, which enabled following measurement and analysis of alveolar mucosa thickness in edentulous patients as studied by Januário et al ${ }^{27}$. Lately, a study in 2015 confirmed the accuracy of using CBCT scans in measuring the thickness of edentulous alveolar mucosa under the fitting surface of a denture ${ }^{24}$.

Bone loss around implants can be reported in four different ways, mesially, distally, buccally and lingually. Comparatively studies stated separate measurements and others showed combined numbers per single implant. Stating bone loss separately appears more accurate, as measurements between sites can vary. The reason for such disparity might be the shape of alveolar ridge. Sometimes implants are placed on ascending bone crest therefore different implant-abutment junction position mesiodistally or buccolingually in relation to the bone level may occur. Thus, bone loss also can differ between mesial and distal sites. On the other hand, the combined measurement is more easy to understand, to relate and to compare with outcomes of other studies. Stastically, reporting of separate measurements would be more precise. Therefore, the decision to measure bone loss separately if correlation is significant, the mean bone loss per implant can be counted, but if correlation would be unreliable - study should report split measurements 
of all aspect sites of the implant. It appeared that Pearson's correlation coefficient showed significant strong correlation between the mesial, distal,buccal and lingual sites, therefore the mean bone loss per implant was calculated ${ }^{36}$.

In the present study, to measure the soft tissue thickness with cone beam, a denture duplicate was made by using barium titanate polymethyle methacrylate (TiO3/ PMMA)composite because of its potential to enhance radiopacity and enables rapid radiographic detection and had a similar radio opacity and density to that of the alveolar ridge bone. This may increase the effective measurement accuracy $^{37}$. The boundaries of the mucosal surfaces are identified easily because the mucosal tissues density are significantly different from that of the air or/and saliva, which exists in the small and dark spaces. The denture duplicate was lined at every measurement with radiopaque mixture of impression material (alginate and barium titanate) to detect the possible changes in soft tissue thickness.

The results of our study revealed significant bone height loss around the implant for the two groups during follow up period. The amount of periimplant bone loss agrees with the findings obtained by Bryant and Zarb ${ }^{38}$, Nandal et $\mathrm{al}^{39}$ who studied the marginal bone level changes in the 1st year and suggested that the initial marginal bone level change occurred not more than 1-1.5 mm is accepted and it considered as an adaptation of the peri-implant bone to the occlusal load., periosteal reflection, implant osteotomy and the stress factors from bone strain at the implant insertion and stresses applied by the prosthesis. However in the present study, the amount of marginal bone loss was ranged from 0.5 to $0.6 \mathrm{~mm}$ which was less as compared with many researches.

With correlation with the marginal bone loss, there was significant decrease in flabby tissues thickness during the follow up period of both groups. This result was due to the use of implant retained overdenture will minimize the undesirable forces transmitted to the anterior mandibular tissues, improves proprioception and reduces trauma to the underlying tissues, thereby reduces bone resorption, this is in agreement with Ibrahim etal ${ }^{40}$ and Tolstunov ${ }^{41}$.

Also the explanation of little bone loss with decreased soft tissues thickness was based on the studies carried by Berglundh \& Lindhe ${ }^{42}$. as the entire contact length between the implant, the epithelial and the connective tissue portions is defined as "the biological width" If the thickness of the peri-implant mucosa was reduced bone resorption occurred to reestablish the mucosal dimension that was required for protection of the underlying tissues. Also as reported with Glaser et $\mathrm{al}^{43}$, a peri-implant seal around implants in humans is about $4 \mathrm{~mm}$. Apparently, the width of thick mucosal tissues is sufficient for formation of peri implant tissues without or minimal bone resorption. Implants in the thick tissue pattern had significantly less bone loss, compared to thin tissue. In other words, vertical distancing of microgap away from the bone crest can reduce marginal bone loss only if thick mucosal tissues are present at a time of implant placement.

When comparing between the two groups as regards to marginal bone loss and flabby tissues thickness, there was little loss of marginal bone loss and more decrease in flabby tissues thickness with patients received implant retained overdenture that constructed from selective pressure technique than those overdenture constructed from conventional functional impression technique. As the provision of implants brings enormous benefits to edentulous patients. However, a poorly fabricated denture does not become ideal just because it is implant retained. When a patient is dissatisfied with the denture and examination reveals a poorly constructed prosthesis, improvements must made by constructing a new prosthesis and/or providing care for the predisposing oral problems.

The muco-displacive impression technique exhibits excellent retention of the denture during the function, due to the displacement of vascular 
contents of the blood vessels into the interstitial spaces. Nevertheless, when at rest, retention and stability are affected, and the denture becomes loose because blood re-enters the tissues altering its contours. According to the theories of impression making, muco-displacive impression techniques results in an unretentive and unstable denture. Selective pressure or minimally displacive impression techniques overcome these limitations as during impression, part of the tissues that were taken under pressure are stress bearing area and can withstand pressure and not rebound, While the other areas which are flabby were taken under rest, this is in line of studies carried by Khinnavar et al ${ }^{17}$. Clearly, an impression technique is required which will compress the non-flabby tissues to obtain optimal support, and, at the same time, will not displace the flabby tissues. So the used selective pressure impression technique in this study removed any soft tissue folds and smoothies them over the mandibular bone; this reduces the potential for discomfort arising from the "atrophic sandwich the creased mucosa lying between the denture base and the mandibular bone.

In addition, the displaceable tissue was recorded with mucostatic impression material where the overdenture is implant retained and other areas in compressive impression material. This helped in achieving the properly retentive and stable denture, which provides flabby tissue improvement. These results were in agreement with Vohra ${ }^{19}$.

\section{CONCLUSION}

Fibrous ridges deceive a prosthodontic defy for the fulfillment of stable and retentive dental prostheses. Implants could be successfully placed in flabby alveolar ridges. But recording the displaceable tissues under mucostatic condition where the overdenture is implant retained and other areas in compressive impression materials is better to preserve the implant and to help in achieving the properly retentive and stable denture.

\section{REFERENCES}

1. Glossary of Prosthodontic Terms. J Prosthet Dent 2005;94:10-92.

2. Lynch CD, Allen PF. Management of the flabby ridge: Using contemporary materials to solve an old problem. $\mathrm{Br}$ Dent J. 2006;200: 258-261

3. Hazari P, Yadav M, Gaikwad A, Singh V, Somkuwar K: Internally weighted dentures: A treatment modality for severly resorbed mandibular ridge : A case report.Journal of Evolution of Medical and Dental Sciences.2014; 3: 10374-10378.

4. Sabarigirinathan1 C, Vinayagave K, Rupkumar $P$, Gandhimathi J, Bhuvaneswari S, BipinchandarL, Making the Unstable Stable-A Case Series of Management of the Flabby Ridge. Journal of Dental and Medical Sciences 2015; 14: 67-71.

5. Mishal M, Souza D, Sabita M. Ram, Bhanushali K : Management of atrophic mandibular ridges with Mini Dental Implant system -A case report.The Journal of Indian Prosthodontic Society. 2005; 5 : 158-160.

6. Massad J,Lobel W: Building the edentulous impressions - a layering technique using multiple viscosities of impression materials. Compendium 2006; 27:446-451.

7. Kumar P, Singh1k, KumarA, KhattarA, GoelR, Preet H.: Impression techniques for hypermobile alveolar mucosa. Int J Health Allied Sci 2012; 1:255-257.

8. MacEntee MI. Identifying the Problem. The Complete Denture: A Clinical Pathway. 2nd edtion,Chicago, Quintessence. 2014; 1-7

9. Sudhir P, Rathod N, Rathod C, Naitam D, Choudhury G K: Management of Flabby Maxillary Ridge- A Case Report. Journal of Health Sciences.2013;1: 83-86.

10. Crawford RWI, Walmsley AD. A review of prosthodontic management of fibrous Ridges. Br Dent J. 2005; 199: 715-719.

11. Poonam K. Khinnavar, B. H. Dhanya Kumar, H. R. Shivakumar, D. B. Nandeeshwarl: Multidisciplinary approach in rehabilitating flabby ridge in completely edentulous patient European Journal of Prosthodontics. 2015; 3: 2 51-55.

12. Basker RM, Davenport JC. Prosthetic treatment of the edentulous patient. Oxford: Blackwell, 2002. 4th ed. pp 286-289. 
13- Chaturvedi M, Chaturvedi S, Ahmad N, Bashir T: Revisiting Hypermobile Mucosa In Edentulous Foundation - Pathology And Management.Journal of Dental and Medical Sciences.2013; 4: 30-35

14. Deol S, Pathak S, Arora A.Fluid retained denture a relief for flabby ridge: A case report J Dent Specialities, 2015;31:109-111

15. Allen PF and McCarthy S. Complete Dentures from Planning to Problem Solving, Quintessence Publishing, London, UK, 2003.

16. Bindhoo YA, Thirumurthy VR, Kurien A. Complete mucostatic impression: A new attempt. J Prosthodont 2012;21:209-214

17. Khinnavar PK, Dhanya Kumar BH, Shivakumar HR, Nandeeshwar DB. Multidisciplinary approach in rehabilitating flabby ridge in completely edentulous patient. Eur J Prosthodont 2015;3:51-55.

18. Watson RM, Davis DM, Forman GH, Coward, T Considerations in design and fabrication of maxillary implant-supported prostheses. Int J Prosthodont. 1991; 4:232-239.

19. Vohra FA, Implant Retained Overdenture as first choice of care for edentulous mandibles:APresentation of two cases. J Pak DentAssoc. 2012; 21: 182 - 187

20. Hermann JS, Buser D, Schenk RK, Cochran DL. Crestal bone changes around titanium implants. A histometric evaluation of unloaded non-submerged and submerged implants in the canine mandible. J Periodontol.2000;71:1412-1424.

21. Berglundh T, Abrahamsson I, Welander M, Lang NP, Lindhe J. Morphogenesis of the periimplant mucosa: an experimental study in dogs. Clin Oral Implants Res.2007; 18:1-8.

22. Iacono VJ.Dental implants in periodontal therapy. J Periodontol.2000;71:1934-1942

23. Wara-aswapati N, Pitiphat W, Chandrapho N, Rattanayatikul C, Karimbux N. Thickness of palatal masticatory mucosa associated with age. J Periodontol. 2001; 72:1407-1412.

24. Dong J, 1 Zhang F, Hui Wu G, Zhang W and Jian Yin. Measurement of Mucosal Thickness in Denture-bearing Area of Edentulous Mandible. Journal List Chin Med J.2015; $128: 32015-3201$
25. Suomalainen A, Vehmas T, Kortesniemi M, Robinson S, Peltola J. Accuracy of linear measurements using dental cone beam and conventional multislice computed tomography. Dentomaxillofac Radiol. 2008;37:10-17.

26. Barriviera $\mathrm{M}$, Duarte $\mathrm{WR}$, Januário $\mathrm{AL}$, Faber $\mathrm{J}$, Bezerra AC. A new method to assess and measure palatal masticatory mucosa by cone-beam computerized tomography. J Clin Periodontol. 2009;36:564-568.

27. Januário AL, Barriviera M, Duarte WR. Soft tissue conebeam computed tomography: A novel method for the measurement of gingival tissue and the dimensions of the dentogingival unit. J Esthet Restor Dent. 2008;20:366-373

28. Ueno D, Sekiguchi R, Morita M, Jayawardena A, Shinpo S, Sato J, et al. Palatal mucosal measurements in a Japanese population using cone-beam computed tomography. J Esthet Restor Dent. 2014; 26:48-58.

29. Umesh Y, Reddy V, and Hosi R : A Single Step Impression Technique of Flabby Ridges Using Monophase Polyvinylsiloxane Material: A Case Report in Dentistry. 2014; Article ID 104541, 6 pages

30. Jayaprakash MB, Sahu K, Khan M, Khoriya S, Jadhav S, Kendre B, Lukaram A. Management of Flabby Ridge Cases: A Challenge in Clinical Practice. Int J Adv Health Sci 2014; 15: 32-37.

31. Krishna Prasad D., Mehra D \& Pranughs A.Prosthodontic management of compromised ridges and. Nitte University Journal of Health Science.2014;4: 2249-7110

32. Arora1 P, Arora V, Singh SP and Saini J. Case Report Prosthodontic Management of Flabby Ridge - From Modified Impression to Denture British Bliomedical Bulletin. 2015;4:508-521

33. Scarfe WC, Farman AG, Sukovic P. Clinical applications of cone-beam computed tomography in dental practice. J Can Dent Assoc. 2006;72:75-80.

34. Ghoneima A, Kula K. Accuracy and reliability of conebeam computed tomography. for airway volume analysis. Eur J Orthod.2013; 35: 256-261.

35. Abdelhamid AM, Assaad N, Weheda AH. Evaluation of Mucosal Displacement and Denture Settlement for Direct Vs. Indirect Attachment Incorporation in Implant Assisted Mandibular Overdentures.J Dent Health Oral disorder and therapy.2016; 5: 00146.

36. Linkevicius T, Pros D, Apse P, Habil M, GrybauskasS, Puisys A. The Influence of Soft Tissue Thickness on Crestal Bone Changes Around Implants: A 1-Year Prospective Controlled Clinical Trial The International journal of oral \& maxillofacial implants;2009 24: 712-719. 
37. Nidal W., Elshereksi L, Saied H, Mohamed A, Arifin1 A and Zainal A.M. Ishak1 P. Evaluation of the Mechanical and Radiopacity Properties of Poly(methyl methacrylate)/ Barium Titanate-denture Base Composites. Polymers \& Polymer Composites.2016;. 24. 5-10.

38. Bryant SR, Zarb GA. Crestal bone loss proximal to oral implants in older and younger adults. J Prosthet Dent. 2003;89:589-597.

39. Nandal, Shikha, Pankaj Ghalaut, and Himanshu Shekhawat. "A Radiological Evaluation of Marginal Bone around Dental Implants: An in-Vivo Study." National Journal of Maxillofacial Surgery 2014; 2:126-137.

40. Ibrahim T O and Ibrahim RO. Evaluation of Two Treatment
Modalities for Patients with Combination Syndrome Suffering From Narrow Anterior Maxilla. Journal of Dental and Medical Sciences. 2013; 10: 2199-2210.

41. Tolstunov, L. Combination syndrome: classification and case report. J Oral Implantology 2007;33: 139-151.

42. Berglundh $\mathrm{T}$, Lindhe J. Dimension of the periimplant mucosa. Biological width revisited. J Clin Periodontol. 1996; 23:971-973

43. Glaser R, Schupbach P, Gottlow J, Hammerle CH. Periimplant soft tissue barrier at experimental one-piece mini-implants with different surface topography in humans: A light microscopic overview and histometric analysis. Clin Implant Dent Relat Res.2005; 7:544-551 